\title{
LOW AND HIGH FREQUENCY QUASI-PERIODIC OSCILLATIONS IN 4U1915-05
}

\author{
L. Boirin ${ }^{1}$, D. Barret ${ }^{1}$, J. F. Olive ${ }^{1}$, J. E. Grindlay ${ }^{2}$ and P.F. Bloser ${ }^{2}$ \\ ${ }^{1}$ CESR, 9 Avenue du Colonel Roche, 31028 Toulouse Cedex 04, France (Laurence.Boirin@cesr.fr) \\ ${ }^{2}$ Harvard Smithsonian Center for Astrophysics, 60 Garden Street, Cambridge, MA 02138, USA
}

\begin{abstract}
The burster and dipper Low Mass X-ray Binary (LMXB) 4U1915-05 (also known as XB1916-053) was observed by the Rossi X-ray Timing Explorer (RXTE) 19 times for a total exposure of 140 ks between 1996 February and October. Here we report on the discovery of Low Frequency (10-40 Hz) Quasi-Periodic Oscillations (LFQPOs) from 4U1915-05. The properties of the LFQPOs are related to the presence of the High Frequency QPOs (HFQPOs) detected simultaneously. We have observed a correlation between the LFQPO frequency and source count rate, as well as a correlation (linear) between the LFQPOs and HFQPOs. Both results cannot be explained by a Beat Frequency Model (BFM). They are also hardly compatible with predictions from the inner disk precession model.
\end{abstract}

\section{INTRODUCTION}

Prior to RXTE, GINGA observations had shown that 4U1915-05 displayed very low variability at frequencies below $1 \mathrm{~Hz}$ (Yoshida, 1992). The same observations suggested also that 4U1915-05 could be an Atoll source. 4U1915-05 was observed by RXTE both in a high intensity/soft state $\left(\mathrm{L}_{2-20 \mathrm{keV}} \sim 1.4 \times 10^{37}\right.$ $\left.\operatorname{ergs~s}^{-1}, 9.3 \mathrm{kpc}\right)$ and a low intensity/hard state $\left(\mathrm{L}_{2-20 \mathrm{keV}} \sim 3.2 \times 10^{36} \mathrm{ergs} \mathrm{s}^{-1}\right)$. A correlated timing and spectral study confirmed the Atoll nature of the source (Boirin et al., 1999). In the low intensity regime, HFQPOs were detected between 600 and $1000 \mathrm{~Hz}$ (Barret et al., 1999, Boirin et al., 1999). Their frequency positively correlates with the count rate, except for an observation performed on October 29th when a $600 \mathrm{~Hz}$ QPO was detected. For two observations, a second marginally significant signal was found at a frequency $350 \mathrm{~Hz}$ lower than the main HFQPO. Applying the "shift and add" technique (Mendez et al., 1998) revealed twin HFQPOs separated by $355 \mathrm{~Hz}$ at 650 and $1005 \mathrm{~Hz}$. In this paper we report on the discovery of LFQPOs in 4U1915-05, and relate their properties to those of HFQPOs.

\section{DISCOVERY OF LFQPOs}

We used the high time resolution Proportional Counter Array (PCA) data (122 $\mu$ s resolution and 0.95 $\mu$ s for the GoodXenon (G) mode). Power Density Spectra (PDS) $\left(3 \times 10^{-3}-100 \mathrm{~Hz}\right)$ were computed in the 5-30 keV range (for all details about the observation and the data analysis, see Boirin et al., 1999). The source displays very low variability below $1 \mathrm{~Hz}$. The Root Mean Square (RMS) integrated up to $1 \mathrm{~Hz}$ ranges between $\sim 3$ and $6 \%$ independently of the spectral/intensity state. Above $1 \mathrm{~Hz}$, in the high intensity state, 4U1915-05 does not display any variability either. On the other hand, at lower 
intensities, the source displays larger variability, with RMS ranging from roughly 8 to $28 \%$ as the count rate decreases. A careful inspection of the LF PDS revealed the presence of broad QPO-like features. Figure 1 (left) shows the LFQPOs in order of decreasing count rate from top to bottom. Parameters of the LFQPOs are given on the plot (see also Table 1). With the exception of the October 29th observation, there is a clear correlation between the LFQPOs and count rate. LFQPOs were detected in five observations. In four of them, HFQPOs were detected simultaneously (above the $5 \sigma$ level) (Barret et al., 1999, see Figure (1). Figure 2 is a plot of the LFQPO frequency against the HFQPO frequency.
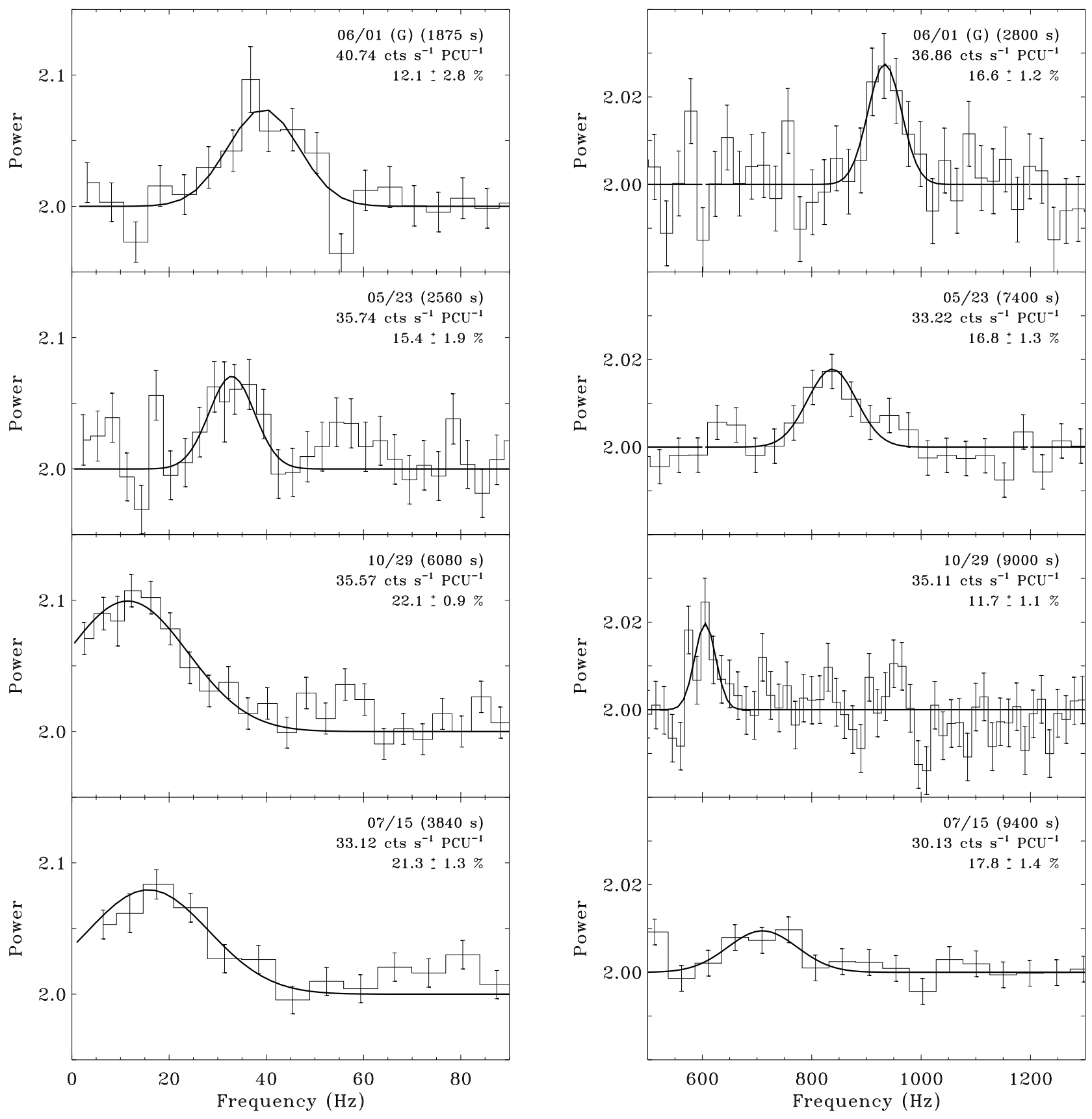

Figure 1: PDS for the observations displaying both LFQPOs (left) and HFQPOs (right). The observation date, exposure time, 5-30 keV count rate before background subtraction and RMS are indicated. For the LFQPOs, the RMS is computed in the $3 \times 10^{-3}-100 \mathrm{~Hz}$ range; the dipping parts of the light curves were filtered out. 


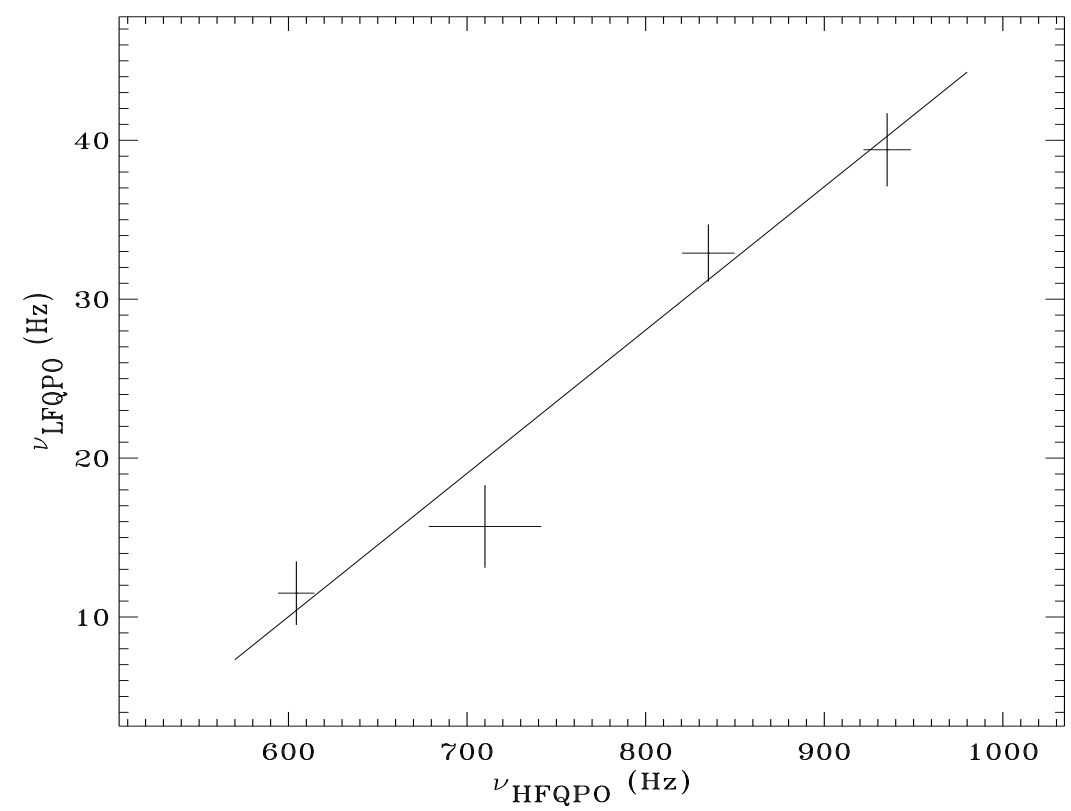

Figure 2: LFQPO versus HFQPO frequency and linear fit.

\section{DISCUSSION}

The main results of this paper can be summarized as follows: we have observed a positive correlation of the LFQPOs with the count rate as well as a correlation between the LFQPOs and HFQPOs. 4U191505 is the third Atoll source, to date, to display such a behaviour; the other two being 4U0614+091 (Ford, 1997) and 4U1728-34 (Ford \& van der Klis, 1998). Such correlations are already well known in Z sources (eg Sco X-1, van der Klis et al., 1997), thus suggesting that similar processes are at work in both kinds of LMXBs. The origin of LFQPOs in Atoll sources is unclear. The magnetospheric BFM (Alpar et al., 1985) long used to explain the Horizontal Branch (HB) QPOs in Z sources cannot be safely extrapolated to Atoll sources, whose magnetic field and accretion rate are much lower than the inferred value for $\mathrm{Z}$ sources. Furthermore, the BFM, if used to explain the LFQPOs cannot account at the same time for the presence of HFQPOs (van der Klis et al., 1997). Note also that in any of such models one would expect a strict correlation between the LFQPOs and count rate. As said earlier, the October 29th observation departs from such a correlation. It is interesting to note that the $600 \mathrm{~Hz}$ HFQPO is also outside the correlation between HFQPOs and count rate, but surprisingly (as shown in Figure 2) falls right on the correlation between LFQPOs and HFQPOs.

Stella and Vietri (1998) developed the Lense-Thirring (LT) inner disk precession model that might account for LFQPOs when HFQPOs are present. This model has been succesfully applied to three sources (4U1728-34, 4U0614+091 and KS1731-260, Stella \& Vietri, 1998) but might have some problems. First, in 4U1735-44, Wijnands et al. (1998) computed a precession frequency greater by a factor of 2 than the LFQPO observed. Second, in 4U1728-34, although the LFQPO frequency varies as the squared of the HFQPO frequency as expected for the precession frequency, the parameters derived for the Neutron Star (NS) are not allowed by classical models (Ford et al., 1998). Finally, a similar conclusion was also reached when the LT model was tried on Z sources (Stella \& Vietri, 1998, Jonker et al., 1998). For 4U1915-05, we assumed a NS spin frequency of $355 \mathrm{~Hz}$ and the keplerian frequency equal to the observed HFQPO frequency. We used the same parameters as Stella and Vietri for the NS (NS mass $\mathrm{M}_{\mathrm{O}}=1.97 \mathrm{M}_{\odot}$, moment of inertia $\left.\mathrm{I}=1.98 \mathrm{M}_{\mathrm{o}} 10^{45} \mathrm{~g} \mathrm{~cm}^{2}\right)$. The results are listed in Table 1. The precession frequency matches the LFQPO frequency for the July 15th observation. For the October 29th, it would be also the case if the $\sim 600 \mathrm{~Hz}$ HFQPO were associated with the inner-disk keplerian frequency. For the remaining two 


\begin{tabular}{|llll|}
\hline Date & $\begin{array}{l}\nu_{H F Q P O} \\
(\mathrm{~Hz})\end{array}$ & $\begin{array}{l}\nu_{L F Q P O} \\
(\mathrm{~Hz})\end{array}$ & $\begin{array}{l}\nu_{\text {precession }} \\
(\mathrm{Hz})\end{array}$ \\
\hline \hline $05 / 23$ & $835.1_{-14.2}^{+14.7}$ & $32.9 \pm 1.8$ & 18.1 \\
\hline $06 / 01(\mathrm{G})$ & $935.2 \pm 13.3$ & $39.4 \pm 2.3$ & 22.5 \\
\hline $07 / 15$ & $710.0_{-30.7}^{+31.5}$ & $15.7 \pm 2.6$ & 13.2 \\
\hline $10 / 29$ & $604.4_{-10.2}^{+9.8}$ & $10.7 \pm 1.5$ & 9.6 \\
& $604.4+355$ & & 23.7 \\
\hline
\end{tabular}

Table 1: Parameters of High and Low Frequency QPOs detected simultaneously from 4U1915-05. $\nu_{\text {precession }}$ is the expected frequency of the inner-disk precession model (Stella \& Vietri, 1998) assuming that 4U1915-05 contains a $355 \mathrm{~Hz}$ rotating NS.

observations (May 23rd and June 1st), the precession frequency is lower by about a factor of $\sim 2$ than the QPO frequency observed. Since the NS parameters used are supposed to yield precession frequencies close to the maximum values allowed in classical NS models, it is unlikely that the LT model can be pushed up to match our LFQPO. Note however, that for the latter two observations, the LFQPO frequency would roughly match the precession frequency if the HFQPO observed were the lowest of a twin pair separated by $355 \mathrm{~Hz}$, or the highest but with a NS spin at $\sim 2 \times 355 \mathrm{~Hz}$. In any case, the LFQPO does not seem to follow the quadratic dependency on the HFQPO, predicted in the LT interpretation (Figure 2). Excluding the peculiar October 29th observation, a fit with a powerlaw gives an index of 3.4 (reduced $\left.\chi^{2}=3.5\right)$. For indication, a simple linear fit yields a lower reduced $\chi^{2}(1.3)$.

\section{CONCLUSIONS}

Our study of the timing properties led to the discovery of LFQPOs. It further revealed a positive correlation between the LFQPOs and count rate, and a correlation between the LFQPOs and HFQPOs. We have shown that our results are hardly compatible with models that have been put forward to explain LFQPOs in Atoll sources. In any case, more observations are needed to better constrain the dependency rules of LFQPOs versus HFQPOs, which in turn might put more stringent constraints on the models.

We thank J. Swank and A. Smale for their support to this project, as well the RXTE/GOF team for his assistance in the data reduction and analysis.

\section{REFERENCES}

Alpar, M.A., \& Shaham, J., 1985, Nature, 316, 239

Barret, D., et al., 1999, Astr. Lett. and Comm., in press

Boirin, L., et al., 1999, A\&AA, in preparation

Ford, E. C., 1997, PhD Thesis, Columbia University

Ford, E. C., \& Van Der Klis, M., 1998, ApJL, 506, L39

Jonker, P.G., et al., 1998,ApJL, 499, L191

Mendez, M., et al., 1998, ApJL, 494, L65

Stella, L., \& Vietri, M., 1998, ApJL, 492, L59

Van der Klis, M., Wijnands, R., Horne, K. and Chen, W., 1997, ApJL, 481, L97

Wijnands, R., et al., 1997, ApJL, 490, L157

Wijnands, R., et al., 1998, ApJL, 495, L39

Yoshida, K., 1992, PhD Thesis, Tokyo University 